\title{
Aspectos clínicos e demográficos de pessoas com deficiência atendidas em uma clínica-escola de Odontologia
}

Eduarda Tomé Ferreira da Silva*; Elizabeth Louisy Marques Soares da Silva-Selva**; Thuanny Silva de Macêdo***; Millena Mirella Silva de Araújo***; Paulo Cardoso Lins Filho***; Camilla Siqueira de Aguiar***; Márcia Maria Vendiciano Barbosa Vasconcelos****; Arnaldo de França Caldas Júnior*****

* Graduada em Odontologia, Universidade Federal de Pernambuco

** Mestre em Saúde da Criança e do Adolescente, Aluna do Programa de Pós-Graduação em Odontologia, Universidade Federal de Pernambuco

*** Mestre em Odontologia, Aluno(a) do Programa de PósGraduação em Odontologia, Universidade Federal de Pernambuco

**** Doutora em Nutrição, Professora do Departamento de Clínica e Odontologia Preventiva, Universidade Federal de Pernambuco

***** Pós-Doutor em Odontologia, Professor do Departamento de Odontologia Social, Universidade de Pernambuco, Professor do Departamento de Clínica e Odontologia Preventiva, Universidade Federal de Pernambuco

Recebido: 26/11/2020. Aprovado: 13/11/2021.

\begin{abstract}
RESUMO
O objetivo deste artigo foi descrever as características clínicas e demográficas de pessoas com deficiência atendidas em uma clínica-escola de Odontologia do Nordeste brasileiro, relacionando estes perfis às necessidades acumuladas e aos tratamentos realizados no período de março a julho de 2019. Tratou-se de um estudo de análise de banco de dados no qual foram avaliados os prontuários em relação a sexo, idade, diagnóstico médico, uso de medicamentos, motivo da consulta e tratamentos realizados. Foi utilizada uma amostra de conveniência de 55 prontuários, referentes aos indivíduos em atendimento neste período. Os testes Qui-quadrado e exato de Fisher foram utilizados para verificar associação entre as variáveis estudadas. Foi adotado o intervalo de confiança de $95 \%$ e a margem de erro de 5\%. Verificou-se que a maioria dos pacientes era do sexo masculino $(52,7 \%)$ e possuía entre 20 e 59 anos $(54,5 \%)$. As patologias de base de maior prevalência foram as doenças sistêmicas $(34,5 \%)$ e deficiência intelectual $(32,7 \%)$. Em relação às medicações, $80 \%$ faziam uso contínuo de algum fármaco. A respeito das consultas odontológicas, a procura de $60 \%$ da amostra foi por motivo de dor e os procedimentos mais realizados, os restauradores $(63,6 \%)$. Além disso, a estabilização física e sedação medicamentosa foram amplamente utilizadas nos pacientes com deficiência intelectual. $\mathrm{O}$ atendimento odontológico incluiu pacientes com diversas necessidades especiais e, apesar da clínica-escola em questão priorizar a conduta preventiva, a maior parte dos procedimentos executados foi curativo, podendo estar relacionado com a procura tardia pelo tratamento odontológico e dificuldades de acesso.
\end{abstract}

Descritores: Pessoas com Deficiência. Saúde Bucal. Assistência Odontológica. 


\section{INTRODUÇÃO}

Segundo a Classificação Internacional de Funcionalidade, Incapacidade e Saúde $^{1}$, as deficiências podem estar associadas à estrutura ou à função do corpo e correspondem a um desvio do que é geralmente aceito como padrão biomédico normal. As deficiências podem ser temporárias ou permanentes; progressivas, regressivas ou estáveis; intermitentes ou contínuas. $\mathrm{O}$ desvio em relação ao modelo baseado na população, e geralmente aceito como normal, pode ser leve ou grave, variando ao longo do tempo e podendo afetar as funções intelectuais, sensoriais, neuromusculoesqueléticas, endócrinas e imunológicas.

Paciente com necessidades especiais (PNE) é a pessoa que apresenta uma ou mais limitações, temporárias ou permanentes, de ordem intelectual, física, sensorial, emocional, de crescimento ou médica, que o impeça de ser submetido a uma situação odontológica convencional. É importante destacar que esse conceito é amplo e abrange diversos casos que requerem atenção odontológica diferenciada ${ }^{2}$.

Saber identificar o PNE é essencial para adequar o seu tratamento de acordo com suas peculiaridades $^{3}$. Apesar da necessidade de um acompanhamento odontológico adequado, a Organização Mundial da Saúde (OMS) relata que apenas $3 \%$ da população total de deficientes têm acesso aos tratamentos odontológicos ${ }^{4}$. É necessário mudar o olhar em todos os sentidos e reconhecer a necessidade de os serviços públicos de saúde serem efetivos, permitindo que as ações de saúde alcancem o processo inclusivo dessas pessoas com deficiência ${ }^{5}$.

O deficiente apresenta inúmeros problemas decorrentes de diferentes bases etiológicas com a probabilidade de ocorrência de distúrbios de comunicação, locomoção e sentido, sendo constantes os problemas de ordem médica e odontológica, que comprometem, sobremaneira, seu bem-estar. Esta realidade torna importante a necessidade da atuação de uma equipe multiprofissional integrada e especializada ${ }^{6}$.

Muitas vezes, o fato de não suprir todas as necessidades odontológicas dos PNEs em questão pode não estar diretamente relacionado com as dificuldades inerentes aos serviços ou até mesmo com as necessidades especiais do paciente. Essas dificuldades podem estar relacionadas com os próprios profissionais, e não quanto à técnica odontológica, somando-se à complexidade que exige um atendimento para pessoas com necessidades especiais, desde a abordagem ao paciente ${ }^{5}$.

Os PNEs possuem uma maior prevalência de doenças bucais como cáries, perdas dentárias, periodontites, hábitos parafuncionais e máoclusões. Estas alterações estão frequentemente associadas a um déficit na higiene oral gerada pelas limitações físicas e psíquicas, pelo tipo de dieta, pela dificuldade em mastigar e deglutir ou pelos diversos medicamentos que são ingeridos diariamente ${ }^{7}$.

$\mathrm{O}$ atendimento a pacientes com necessidades especiais, no nível de graduação, proporciona, durante a formação do profissional, mais do que apenas técnicas para os atendimentos preventivos e curativos, mas também experiências com outras ciências, como a das relações interpessoais que vão servir para a formação do aluno como indivíduo. Sendo assim, é de grande importância quantificar e qualificar este grupo de pacientes para caracterizar melhor o serviço de atendimento e oferecer ao aluno um aprendizado de qualidade ${ }^{8}$.

Ainda que algumas instituições não possuam a disciplina na sua matriz curricular, a sua inclusão é muito importante para a formação de um profissional que atue sobre uma nova lógica, voltada para a humanização e a integralidade do cuidado $^{9,10}$

A disciplina de Pacientes com Necessidades Especiais e Odontologia Hospitalar do curso de Odontologia da Universidade Federal de 
Pernambuco possui um corpo docente especializado no atendimento desta população. A clínica recebe crianças e adultos especiais de todo o estado para o atendimento clínico, preventivo e de urgência realizado por estudantes do curso de graduação com auxílio dos professores. Portanto, de acordo com o supracitado, objetivou-se descrever as características clínicas e demográficas dos pacientes atendidos nesta clínica relacionando às necessidades acumuladas e os tratamentos realizados no período de março a julho de 2019.

\section{MÉTODOS}

Trata-se de um estudo de análise de banco de dados, realizado em uma clínica-escola de atendimento a Pacientes com Necessidades Especiais e Odontologia Hospitalar do Nordeste brasileiro. O projeto foi submetido ao Comitê de Ética local e aprovado com parecer $\mathrm{n}^{\mathrm{o}}$ 3.137.501.

A coleta de dados foi realizada por meio de consulta aos prontuários odontológicos dos pacientes de ambos os sexos, atendidos na Clínica de Pacientes com Necessidades Especiais e Odontologia Hospitalar, no período entre março e maio de 2019.

Foram registrados os dados relativos a sexo, idade, região de moradia, diagnóstico médico da necessidade especial de saúde, uso de medicamentos, motivo da consulta, uso de sedação, tratamentos realizados e condições comportamentais dos usuários desta clínica.

As análises descritivas foram apresentadas sob a forma de tabelas com valores absolutos e respectivos percentuais. Os testes Qui-quadrado e exato de Fisher foram utilizados para verificar associação entre as variáveis estudadas. Foi adotado o valor de $\mathrm{p}<0,05$ como estatisticamente significativo, além de erro de $5 \%$ e intervalo de confiança de $95 \%$. Os dados foram analisados com o auxílio do software SPSS versão 23.0 (IBM, Armonk, NY, EUA).

\section{RESULTADOS}

Foram avaliados cinquenta e cinco prontuários referentes aos pacientes em atendimento no período de janeiro a julho de 2019.

Na tabela 1 observou-se que a maioria dos pacientes $(52,7 \%)$ eram do sexo masculino, com $54,5 \%$ destes possuindo entre 20 e 59 anos de idade. Apenas 16,4\% indivíduos residiam no interior do Estado de Pernambuco e sua grande maioria $(58,2 \%)$ utilizou o ônibus como principal meio de transporte para ir à consulta odontológica. Além disso, verificou-se que $56,4 \%$ tinham os cuidados realizados prioritariamente pela mãe.

Quanto às condições de saúde e medicações de uso contínuo, foi verificado que a maioria dos pacientes em atendimento apresentava doenças sistêmicas (34,5\%). Em relação às medicações, a maioria ( $80 \%$ ) fazia uso contínuo de algum fármaco, em que 13 destes $(23,6 \%)$ relataram utilizar antipsicóticos e 11 $(20,0 \%)$ tomavam medicação para diminuir a ansiedade (tabela 2).

Quanto à consulta odontológica e à condição de saúde bucal da amostra estudada, 60\% dos pacientes procuraram a clínica por motivo de dor e 90,9\% dos indivíduos nunca tiveram problemas durante o tratamento odontológico. Foi necessária a estabilização física em $20 \%$ dos pacientes e $72,7 \%$ dos usuários utilizaram sedação medicamentosa em todas as consultas, exceto a inicial. Referente aos procedimentos realizados, $63,6 \%$ foram restauradores e 30,9\% cirúrgicos (tabela 3).

A tabela 4 descreve os resultados da análise relacionando a patologia de base com outras variáveis. Os pacientes com deficiência intelectual possuem o cuidador principal representado pela mãe $(88,9 \%)$, assim como são os que mais utilizaram a estabilização física $(55,6 \%)$ e a sedação medicamentosa durante atendimento odontológico (50\%). Todos os pacientes com doenças sistêmicas mostraram-se cooperativos ao 
tratamento odontológico nesta clínica $(\mathrm{p}<0,01)$, bem como os deficientes físicos e sensoriais.

Tabela 1. Perfil sociodemográfico dos pacientes atendidos na Clínica de Atendimento a Pacientes com Necessidades Especiais

\begin{tabular}{|c|c|c|}
\hline Variáveis & n & $\%$ \\
\hline \multicolumn{3}{|l|}{ Sexo } \\
\hline Masculino & 29 & 52,7 \\
\hline Feminino & 26 & 47,3 \\
\hline \multicolumn{3}{|l|}{ Idade (anos) } \\
\hline $3-19$ & 22 & 40,0 \\
\hline $20-59$ & 30 & 54,5 \\
\hline $60-72$ & 3 & 5,5 \\
\hline \multicolumn{3}{|l|}{ Região de moradia } \\
\hline Recife e Região Metropolitana & 44 & 83,6 \\
\hline Interior & 9 & 16,4 \\
\hline \multicolumn{3}{|l|}{ Acesso à água tratada } \\
\hline Sim & 49 & 89,1 \\
\hline Não & 6 & 10,9 \\
\hline \multicolumn{3}{|l|}{ Frequenta a escola } \\
\hline Sim & 21 & 38,2 \\
\hline Não & 24 & 43,6 \\
\hline Não respondeu & 10 & 18,2 \\
\hline \multicolumn{3}{|l|}{ Trabalha } \\
\hline Sim & 6 & 10,9 \\
\hline Não & 39 & 70,9 \\
\hline Não respondeu/não se aplica & 10 & 18,2 \\
\hline \multicolumn{3}{|l|}{ Transporte para ir ao dentista } \\
\hline Ônibus & 32 & 58,2 \\
\hline Carro particular/táxi & 13 & 23,6 \\
\hline Ambulância & 1 & 1,8 \\
\hline Transporte municipal & 6 & 10,9 \\
\hline Outros & 3 & 5,5 \\
\hline \multicolumn{3}{|l|}{ Quem cuida do(a) paciente } \\
\hline Mãe & 31 & 56,4 \\
\hline Outra pessoa & 24 & 43,6 \\
\hline
\end{tabular}


Tabela 2. Perfil de saúde dos pacientes atendidos na Clínica de Atendimento a Pacientes com Necessidades Especiais

\begin{tabular}{lcc}
\hline Variáveis & n & \% \\
\hline Necessidade especial de saúde & & \\
Deficiência física & 3 & 5,5 \\
Deficiência intelectual & 18 & 32,7 \\
Deficiências sensoriais & 2 & 3,6 \\
Causas múltiplas & 9 & 16,4 \\
Doenças sistêmicas & 19 & 34,5 \\
Gravidez & 3 & 5,5 \\
Câncer & 1 & 1,8
\end{tabular}

\section{Em tratamento atualmente}

$\begin{array}{ccc}\text { Sim } & 42 & 76,4 \\ \text { Não } & 13 & 23,6 \\ \text { Uso de medicação continua } * & 44 & 80,0 \\ \text { Anticonvulsivante } & 9 & 16,4 \\ \text { Antipsicótico } & 13 & 23,6 \\ \text { Ansiolítico } & 11 & 20,0 \\ \text { Antiepiléptico } & 9 & 16,4 \\ \text { Antidepressivo } & 4 & 7,3 \\ * \text { Pacientes poderiam se enquadrar em mais de uma opção de medicação. }\end{array}$

\section{DISCUSSÃO}

De acordo com o perfil clínico e sociodemográfico avaliado neste estudo, verificouse que $\mathrm{o}$ atendimento odontológico incluiu pacientes com diversas necessidades especiais de saúde, faixa etária e necessidades de tratamento, evidenciando assim, a importância de uma melhor assistência odontológica a essa população e suas particularidades $^{5,6}$.

Com relação ao sexo pôde-se observar que o masculino apresentou uma maior prevalência, o que coincide com outros estudos ${ }^{11,12}$. Em contrapartida, outro estudo desenvolvido com uma população semelhante observou que a maioria dos pacientes foram do sexo feminino ${ }^{13}$. Quanto à faixa etária predominante, destacou-se a de 20 a 59 anos de idade (54,5\%), corroborando outras investigações $^{14,15}$.

A maior prevalência dos pacientes era de residente da Região Metropolitana do Recife, onde o mesmo pode ser constatado na literatura analisada $^{9,15}$, na qual a maioria dos pacientes residiam na mesma cidade ou região próxima na qual ocorriam os atendimentos em questão. É valido ressaltar a importância da clínica de referência, na realidade local, o Centro de Especialidades Odontológicas (CEO), pois há casos que extrapolam os limites dos serviços da Atenção Básica e é necessário ter o suporte de um especialista, conforme a política que assegura a assistência integral ao indivíduo em todos os seus níveis de complexidade ${ }^{16}$. 
Tabela 3. Características odontológicas observadas durante anamnese do paciente e tratamentos realizados

\begin{tabular}{|c|c|c|}
\hline Variáveis & $\mathbf{n}$ & $\%$ \\
\hline \multicolumn{3}{|l|}{ Motivo da consulta } \\
\hline Urgência & 33 & 60,0 \\
\hline Rotina/revisão & 22 & 40,0 \\
\hline \multicolumn{3}{|l|}{ Secura na boca } \\
\hline Sim & 17 & 30,9 \\
\hline Não & 38 & 69,1 \\
\hline \multicolumn{3}{|l|}{ Ardência bucal } \\
\hline Sim & 4 & 7,3 \\
\hline Não & 51 & 92,7 \\
\hline \multicolumn{3}{|c|}{ Tem ou teve alguma mancha branca na mucosa bucal } \\
\hline $\operatorname{Sim}$ & 3 & 5,5 \\
\hline Não & 52 & 94,5 \\
\hline \multicolumn{3}{|l|}{ Presença frequente de aftas } \\
\hline Sim & 4 & 7,3 \\
\hline Não & 51 & 92,7 \\
\hline \multicolumn{3}{|l|}{ Halitose } \\
\hline Sim & 24 & 43,6 \\
\hline Não & 31 & 56,4 \\
\hline \multicolumn{3}{|l|}{ Uso de estabilização física } \\
\hline Sim & 11 & 20,0 \\
\hline Não & 44 & 80,0 \\
\hline \multicolumn{3}{|l|}{ Uso de sedação medicamentosa } \\
\hline Não & 44 & 80,0 \\
\hline Sim & 11 & 20,0 \\
\hline 1 consulta & 2 & 18,2 \\
\hline 2 ou mais consultas & 1 & 9,1 \\
\hline Todas as consultas, excluindo a inicial & 8 & 72,7 \\
\hline \multicolumn{3}{|l|}{ Procedimentos realizados* } \\
\hline Diagnóstico & 55 & 100,0 \\
\hline Prevenção & 34 & 61,8 \\
\hline Endodontia & 19 & 34,5 \\
\hline Periodontia & 30 & 54,5 \\
\hline Cirurgia & 17 & 30,9 \\
\hline Dentística & 35 & 63,6 \\
\hline Ortodontia & 1 & 1,8 \\
\hline
\end{tabular}

*Pacientes poderiam se enquadrar em mais de uma opção de procedimento. 
Tabela 4. Tipos de abordagens durante tratamento odontológico segundo necessidade especial de saúde

\begin{tabular}{|c|c|c|c|c|c|c|}
\hline \multirow[b]{2}{*}{ Variáveis } & \multicolumn{6}{|c|}{ Necessidades Especiais de Saúde } \\
\hline & $\begin{array}{c}\text { Deficiência } \\
\text { física } \\
\mathrm{n}(\%) \\
\end{array}$ & $\begin{array}{c}\text { Deficiência } \\
\text { intelectual } \\
\mathrm{n}(\%) \\
\end{array}$ & $\begin{array}{c}\text { Deficiência } \\
\text { sensorial } \\
\mathrm{n}(\%)\end{array}$ & $\begin{array}{c}\text { Causas } \\
\text { múltiplas } \\
\mathrm{n}(\%)\end{array}$ & $\begin{array}{c}\text { Doenças } \\
\text { sistêmicas } \\
\mathrm{n}(\%)\end{array}$ & p-valor \\
\hline \multicolumn{7}{|c|}{ Quem cuida do paciente } \\
\hline Mãe & $2(66,7)$ & $16(88,9)$ & $1(50,0)$ & $7(77,8)$ & $5(26,3)$ & \multirow{2}{*}{$<0,01^{*}$} \\
\hline Outro & $1(33,3)$ & $2(11,1)$ & $1(50,0)$ & $2(22,2)$ & $14(73,7)$ & \\
\hline \multicolumn{7}{|l|}{ Motivo da consulta } \\
\hline Dor & $0(0,0)$ & $12(66,7)$ & $2(100,0)$ & $3(33,3)$ & $12(63,2)$ & \multirow{2}{*}{$0,07 *$} \\
\hline Rotina/revisão & $3(100,0)$ & $6(33,3)$ & $0(0,0)$ & $6(66,7)$ & $7(36,8)$ & \\
\hline \multicolumn{7}{|c|}{ Problemas no tratamento odontológico } \\
\hline Sim & $0(0,0)$ & $3(16,7)$ & $0(0,0)$ & $1(11,1)$ & $1(5,3)$ & \multirow{2}{*}{$0,77 *$} \\
\hline Não & $3(100,0)$ & $15(83,3)$ & $2(100,0)$ & $8(88,9)$ & $18(94,7)$ & \\
\hline \multicolumn{7}{|c|}{ Comportamento durante consultas odontológicas } \\
\hline Cooperativo & $3(100,0)$ & $8(44,4)$ & $2(100,0)$ & $8(88,9)$ & $19(100,0)$ & \multirow{3}{*}{$<0,01^{*}$} \\
\hline Não cooperativo & $0(0,0)$ & $8(44,4)$ & $0(0,0)$ & $1(11,1)$ & $0(0,0)$ & \\
\hline Agressivo & $0(0,0)$ & $2(11,2)$ & $0(0,0)$ & $0(0,0)$ & $0(0,0)$ & \\
\hline \multicolumn{7}{|c|}{ Uso de estabilização física } \\
\hline Sim & $0(0,0)$ & $10(55,6)$ & $0(0,0)$ & $1(11,1)$ & $0(0,0)$ & \multirow{2}{*}{$<0,01^{*}$} \\
\hline Não & $3(100,0)$ & $8(44,4)$ & $2(100,0)$ & $8(88,9)$ & $19(100,0)$ & \\
\hline \multicolumn{7}{|c|}{ Uso de sedação medicamentosa } \\
\hline Sim & $0(0,0)$ & $9(50,0)$ & $0(0,0)$ & $1(11,1)$ & $1(5,3)$ & \multirow{2}{*}{$0,01^{*}$} \\
\hline Não & $3(100,0)$ & $9(50,0)$ & $2(100,0)$ & $8(88,9)$ & $18(94,7)$ & \\
\hline
\end{tabular}

*Teste Exato de Fisher

As doenças sistêmicas foram as mais prevalentes dentre as patologias de base que os pacientes atendidos nesta clínica possuíam. A deficiência intelectual veio em seguida se assemelhando a um outro estudo com $\mathrm{PNE}^{17}$. É válido ressaltar que muitos pacientes com comprometimento sistêmico, assim como as grávidas, não têm impedimento de serem atendidos em outras clínicas que não sejam de pacientes especiais, visto que os procedimentos são realizados igualmente à população normorreativa.

Os resultados de outro estudo atestam o que foi encontrado na presente amostra, mostrando que a maioria dos pacientes fazem uso contínuo de medicações ${ }^{11}$. Os ansiolíticos estão entre os mais utilizados nesta pesquisa, sendo este achado também observado em outro estudo, sendo citado que existe um aumento no número de indivíduos que fazem o uso prolongado destas drogas ${ }^{18}$. Já a classe dos antidepressivos mostrou-se a menos utilizada, assim como em outros trabalhos ${ }^{4,14}$. Vale salientar que alguns desses medicamentos podem, também, atuar promovendo uma hiperplasia gengival que, associada à má higiene bucal, propicia a inflamação e o desenvolvimento da doença periodontal precoce $\mathrm{e}^{19,20}$.

Neste estudos, as mães foram as cuidadoras principais dos pacientes especiais e, consequentemente, são com quem mais se relacionam, mostrando resultados semelhantes em 
outros estudos ${ }^{4,13}$. Constatou-se também em outra investigação que grande parte dos pacientes é dependente de um cuidador para realizar a higiene bucal, sendo este cuidador representado pela mãe em mais de $85 \%$ dos casos ${ }^{4}$. A negligência da saúde bucal dos filhos, pelos pais ou responsáveis, que possa ocorrer, é devido às dificuldades físicas, intelectuais e sociais, e pela priorização de outros cuidados $^{21}$.

Em um estudo desenvolvido com o objetivo de avaliar as condições de saúde bucal dos PNEs, os autores observaram que a maior parte dos pacientes se mostrou cooperativa ao atendimento odontológico e a minoria necessitou de estabilização física para a realização dos procedimentos, o que pôde ser confirmado com os resultados do estudo em questão ${ }^{17}$. A sedação medicamentosa também foi utilizada em $20 \%$ dos pacientes, sendo uma alternativa bastante favorável para os pacientes não colaborativos.

O tratamento odontológico do PNE requer alterações, seja na conduta clínica, como também na compreensão das dificuldades físico/motoras e de acesso aos serviços de saúde ${ }^{22}$. Nesta pesquisa o motivo da consulta mais prevalente foi a dor (dentina exposta), o que difere de um estudo feito em Criciúma - SC/ Brasil, onde o motivo que levou os pais e/ou responsáveis dos pacientes a procurarem assistência odontológica pela primeira vez foi, em grande parte, para uma consulta de rotina $^{13}$.

Dentre as intervenções odontológicas apresentadas neste estudo, observou-se que procedimentos mais executados foram os restauradores e em seguida os preventivos, como em outras pesquisas ${ }^{11,17}$. Muitas vezes a demora na procura de tratamento impede uma atuação mais precoce do profissional e, consequentemente, resulta em necessidades de tratamento curativo acumuladas. A importância de condutas preventivas é indiscutivelmente importante para todos os indivíduos, mas de uma forma especial para estes devido às dificuldades encontradas para os tratamentos odontológicos eletivos ${ }^{23}$. Para tanto, é de grande importância buscar estratégias de promoção à saúde, incentivando, inclusive, os alunos do curso de Odontologia a participarem de projetos de extensão que aproximem essa população e seus cuidadores de uma efetiva prevenção dos agravos bucais.

É importante ressaltar que essas dificuldades são devido à falta de controle e coordenação dos movimentos da mandíbula, dos lábios e da língua que os pacientes com necessidades especiais apresentam, além da existência de muita dificuldade por parte dos familiares e/ou responsáveis em promover uma higiene bucal adequada. Esse quadro é agravado pelos seguintes aspectos: carência de programas de treinamento para cuidadores; profissionais pouco qualificados e despreparados para este tipo de atendimento; custo financeiro; desconhecimento dos pais quanto às possibilidades de tratamento, e priorização do tratamento de outros problemas sistêmicos ${ }^{14}$.

Diante do exposto, depreende-se a importância do atendimento ao PNE durante a graduação pois possibilita quantificar e qualificar este grupo de pacientes, caracterizar melhor o serviço de atendimento prestado, além de oferecer ao aluno de graduação um aprendizado de qualidade e experiências que o nortearão para o acolhimento e atendimento humanizado ao PNE.

\section{CONCLUSÃO}

$\mathrm{O}$ atendimento odontológico incluiu pacientes com diversas necessidades especiais e que a maior parte dos procedimentos executados foi curativo, podendo estar relacionado com a procura tardia pelo tratamento odontológico e dificuldades de acesso.

\footnotetext{
ABSTRACT

Clinical and demographic characteristics of people with disabilities attending a dental
} 


\section{teaching clinic}

The aim of this article was to describe the clinical and demographic characteristics of people with disabilities attending a dental teaching clinic in northeastern Brazil, and to associate these profiles with the cumulative needs and treatments performed from March to July 2019. This was a database study in which sex, age, medical diagnosis, medication use, reason for consultation, and previous treatments obtained from the medical records were evaluated. A convenience sample of 55 records from patients seen during this period was used. The chi-square test and Fisher's exact test were applied to evaluate the association between the variables studied, adopting a $95 \%$ confidence interval and an error margin of $5 \%$. Most patients were male $(52.7 \%)$ and were between 20 and 59 years old (54.5\%). The most prevalent underlying diseases were systemic diseases (34.5\%) and intellectual disability (32.7\%). Regarding medications, $80 \%$ of the patients continuously used some drug. The reason for consultation was pain in $60 \%$ of the sample and restorative procedures were the most frequently performed $(63.6 \%)$. Moreover, patients with intellectual disability commonly required physical restraint and sedatives. The dental consultations comprised patients with different special needs. Although the teaching clinic prioritizes preventive management, most of the procedures performed were curative, a fact that might be related to the late seeking of dental treatment and difficulties of accessing health services.

Descriptors: People with Disabilities. Oral Health. Dental Care.

\section{REFERÊNCIAS}

1. Organização Mundial da Saúde. CIF: Classificação Internacional de Funcionalidade, Incapacidade e Saúde. Tradução Centro Colaborador da Organização Mundial da Saúde para a Família de Classificações Internacionais. São Paulo: Edusp, 2003.
2. Brasil. Ministério da Saúde. Cadernos de Atenção Básica, n. 17. Saúde Bucal. Brasília: Editora do Ministério da Saúde, 2006. 92 p.

3. Campos CC, Frazão BB, Saddi GL, Morais LA, Ferreira MG, Setúbal PCO et al. Manual prático para o atendimento odontológico de pacientes com necessidades especiais. Goiânia: Universidade Federal de Goiás - Faculdade de Odontologia, p. 26-29, 2009.

4. Nasiloski KS, Silveira ERD, César Neto JB, Schardosim LR. Avaliação das condições periodontais e de higiene bucal em escolares com transtornos neuropsicomotores. Rev Odontol UNESP 2015; 44(2):103-7.

5. Fonseca ALA, Azzalis LA, Fonseca FLA, Botazzo C. Análise qualitativa das percepções de cirurgiões-dentistas envolvidos nos atendimentos de pacientes com necessidades especiais de serviços públicos municipais. Rev Bras Crescim Desenv Hum. 2010; 20(2):208-16.

6. Marta SN. Programa de assistência odontológica ao paciente especial: uma experiência de 13 anos. Rev Gaúch Odontol 2011; 59(3):379-85.

7. Baumgarten A, Monteiro APS, Petersen RC. Curso de Capacitação para CirurgiõesDentistas no Atendimento à Pessoa com Deficiência e Necessidades Especiais do Estado do Rio Grande do Sul: Perfil do Cirurgião-Dentista, Trajetória no Serviço e Cobertura dos Municípios. Rev Fac Odontol Porto Alegre 2013; 54(1-3):19-23.

8. Silva ZCM, Pagnoncelli SD, Weber JBB, Fritscher AMG. Avaliação do perfil dos pacientes com necessidades especiais da Clínica de Odontopediatria da Faculdade de Odontologia da PUCRS. Rev Odonto Ciência 2005; 20(50):313-8.

9. Cipriano OB, Rolim AKA, Alves MASG, Oliveira- Filho AA, Guênes GMT, Figueiredo 
CHMC et al. Odontologia para pacientes especiais: análise da composição curricular das instituições públicas do Brasil. Res Soc Dev. 2020; 9(2): e189922135.

10. Silva TD, Santaella NG, Caminha RDG, Santos PSS. Percepção de estudantes de graduação sobre a importância da disciplina Odontologia para pacientes com necessidades especiais. Rev ABENO 2020; 20(1):26-32.

11. Oliveira ALP, Chagas SKM, Peixoto MOB, Peixoto FB, Cruz VSA, Ferreira SMS et al. Avaliação sociodemográfica e odontológica de pacientes com deficiência atendidos na clínica-escola de uma instituição de ensino superior. Rev AcBO 2017; 6(2): 58-64.

12. Leite T, Leite NIF, Vassen AB, Antonini F, Lessa LS, Sonego FGF. Condição de saúde bucal, estado nutricional e perfil socioeconômico de pacientes com necessidades especiais. Rev Odontol Univ Cid São Paulo 2018; 30(1): 21-32.

13. Crescêncio MCC, Cristiano DP, Simões PW, Sonego FGF. Análise do conhecimento de pais ou responsáveis sobre a saúde bucal dos filhos com necessidades especiais. Rev Odontol Univ Cid São Paulo 2018; 30(2): 14456.

14. Domingues NB, Ayres KCM, Mariusso MR, Zuanon ACC, Giro EMA. Caracterização dos pacientes e procedimentos executados no serviço de atendimento a pacientes com necessidades especiais da Faculdade de Odontologia de Araraquara - UNESP. Rev Odontol UNESP 2015; 44(6): 345-50.

15. Pereira LM, Mardero E, Ferreira SH, Kramer $\mathrm{PF}$, Cogo RB. Atenção odontológica em pacientes com deficiências: a experiência do curso de Odontologia da ULBRA Canoas/RS. Stomatos 2010; 16 (31):92-9.

16. Lawrence H, Sousa LP, Gonçalves FL, Saintrain MVL, Vieira APGF. Acesso à saúde pública pelo paciente especial: a ótica do
Cirurgião-Dentista. Rev Bras Promoç Saúde 2014; 27(2): 190-97.

17. Queiroz FS, Rodrigues MMLF, Cordeiro Junior GA, Oliveira AB, Oliveira JD, Almeida ER. Avaliação das condições de saúde bucal de Portadores de Necessidades Especiais. Rev Odontol UNESP 2014; 43(6): 396-401.

18. Coelho MN. Proposta de intervenção para reduzir o uso indiscriminado de ansiolíticos. Universidade de Minas Gerais. Faculdade de Medicina. Núcleo de Educação em Saúde Coletiva. Governador Valadares, 2015. 28p.

19. Gusmão ES, Cimões R, Coelho RS, Milhomens Filho JA, Santos RL, Sales GCF. Diagnóstico e tratamento do aumento gengival induzido por drogas. Rev Cir Traumatol Buco-maxilo-fac. 2009; 9(1): 59-66.

20. Farook FF, M NIZAM, Nizam MNM, Alshammari A. An update on the mechanisms of phenytoin induced gingival overgrowth. Open Dent J 2019; 13(1):430-5.

21. Menchaca HRM, Silva GR, Alanís GT. Guía para el cuidado de la salud oral en pacientes con necesidad de cuidados especiales de salud en México. Rev Adm. 2011; 68(5): 222-8.

22. Oliveira ALBM, Giro EMA. Importância da abordagem precoce no tratamento odontológico de pacientes portadores de necessidades especiais. Odonto 2011; 19(38): 45-51.

23. Nunes R, Simões PW, Pires PDS, Rosso MLP. Prevalência de alterações bucais em pessoas com deficiência na clínica da Universidade do Extremo Sul Catarinense. Rev Odontol Univ Cid São Paulo 2017; 29(2): 118-28.

\section{Correspondência para:}

Arnaldo de França Caldas Júnior e-mail: caldasjr@ alldeia.com.br Estrada de Aldeia, Km 13, Privê Portal de Aldeia, Aldeia 54783-010 Camaragibe/PE 